\title{
Prediction of clinical outcome using p16INK4a immunocytochemical expression in low-grade squamous intraepithelial lesions and high-risk HPV-positive atypical squamous cells of undetermined significance in patients with and without colposcopic evident cervical disease
}

\author{
ANKICA LUKIC ${ }^{1}$, GIORGIO SBENAGLIA ${ }^{1}$, ELISABETTA CARICO ${ }^{2}$, \\ MATILDE DI PROPERZIO ${ }^{1}$, ENRICO GIARNIERI ${ }^{2}$, ANTONIO FREGA ${ }^{1}$, \\ FLAVIA NOBILI $^{1}$, MASSIMO MOSCARINI ${ }^{1}$ and MARIA ROSARIA GIOVAGNOLI ${ }^{2}$ \\ ${ }^{1}$ Dipartimento Salute della Donna e Medicina Territoriale, UOC Ginecologia, Facoltà di Medicina e Psicologia, \\ Sapienza Università di Roma, 00189 Rome; ${ }^{2}$ Dipartimento di Medicina Clinica e Molecolare, \\ UOD Citopatologia, Facoltà di Medicina e Psicologia, Sapienza Università di Roma, 00161 Rome, Italy
}

Received April 18, 2011; Accepted July 1, 2011

DOI: $10.3892 /$ etm.2011.316

\begin{abstract}
INK4a as a diagnostic marker of a cervical intraepithelial neoplasia of grade $2+(\mathrm{CIN} 2+)$ in atypical squamous cells of undetermined significance (ASC-US) and low-grade squamous intraepithelial lesion (LSIL) cytological samples has been analyzed, but has not yet been included in clinical routine practice. One hundred and ninety-one patients with an abnormal Pap test (84 ASC-US and 107 LSILs) who underwent colposcopy were selected for this study. At enrollment, 96 patients (Group 1) had a positive colposcopy and therefore underwent a cervical biopsy, while 95 (Group 2) had a negative colposcopy and were followed up for up to 1 year. Both groups were tested for p16INK4a using immunocytochemical methods, and the p16INK4a results were correlated with histology or follow-up outcome. In Group 1 ASC-US cases, 82\% of lesions less than CIN2 were p16INK4a-negative and all CIN2 cases were p16INK4apositive ( $\mathrm{p}=0.00044)$. In Group 1 LSIL cases, $71 \%$ of lesions less than CIN2 were p16INK4a-negative and $87 \%$ of CIN2/3 were p16INK4a-positive $(\mathrm{p}=0.00033)$. Seventy-seven percent of Group 2 ASC-US patients with a negative 1-year follow-up (NF-U) were p16INK4a-negative at enrollment, while all
\end{abstract}

Correspondence to: Dr Ankica Lukic, Dipartimento Salute della Donna e Medicina Territoriale, UOC Ginecologia, Facoltà di Medicina e Psicologia, Sapienza Università di Roma, Via di Grottarossa 1035-39, 00189 Rome, Italy

E-mail: luki.anki@tin.it

Key words: p16INK4a, low-grade squamous intraepithelial lesion, atypical squamous cells of undetermined significance, colposcopy, histology, cervical intraepithelial neoplasia, high-risk human papillomaviruses patients with positive follow-up (PF-U) were p16INK4apositive $(\mathrm{p}=0.00113)$. In Group 2 LSIL cases, $83 \%$ of patients with NF-U were p16INK4a-negative, while $65 \%$ of patients with PF-U were p16INK4a-positive at enrollment $(\mathrm{p}=0.0014)$. In fact, $39 \%$ of the positive p16INK4a LSIL patients had CIN2+ histological lesions. The positive predictive value of p16INK4a for CIN2+ was $50 \%$ in ASC-US and 52\% in LSIL cases; the negative predictive value was 100 and 94\%, respectively. In conclusion, in our patients, a negative p16INK4a appears to be a marker of the absence of CIN3, while a positive p16INK4a can be correlated with the presence of histological CIN2+ found at enrollment or during the subsequent follow-up. Thus, its clinical predictive value is independent from the colposcopic aspect at enrollment.

\section{Introduction}

It has been proven that human papillomaviruses (HPVs) are strongly associated with pre-neoplastic and neoplastic lesions of the uterine cervix (1-3).

According to present guidelines, patient treatment and followup vary according to the classification of the lesion (4-8). Patients with high-grade squamous intraepithelial lesions (HSILs) are referred for colposcopy and, when a cervical intraepithelial neoplasia of grade 2 or more is histologically confirmed (CIN2+), the lesion is treated with excisional procedures. However, the management of patients with low-grade squamous intraepithelial lesions (LSILs) and atypical squamous cells of undetermined significance (ASC-US) is still controversial. Therefore, the identification of patients that have CIN2+ lesions beneath a LSIL cytological superficial lesion is an important challenge in order to avoid under diagnosis and treatment.

The aim to identify a specific triage test for women with ASC-US and LSILs has become increasingly crucial. The introduction of a biomarker that detects a CIN2+ cervical 
pathology would be very useful in the management of women who have low-grade or undefined lesions.

According to the ALTS study (9), in the case of an ASC-US result, all international guidelines, including those of the Italian Society of Colposcopy, agree on the use of an HPV DNA test to decide whether or not colposcopy is indicative. However, in the case of LSILs, an HPV test is not as specific; therefore, novel markers are required.

The p16INK4a protein has a negative regulatory role in the cellular cycle of eukaryote cells by activating their arrest during the course of differentiating processes $(10,11)$. p16INK4a overexpression indicates inactivation of cellular cycle control mediated by the E7 oncoprotein of high-risk human papillomaviruses (HR-HPVs) (12-18). In normal Pap smears, the p16INK4a protein is not evident by immunocytochemistry, with the exception of some normal cells where it is physiologically expressed (16).

\section{Materials and methods}

From June 2008 to June 2009, 191 patients, 107 with LSILs and 84 with HR-HPV-positive ASC-US, were enrolled in a study at the Colposcopy and the Low Genital Tract Pathology Unit of the Dipartimento Salute della Donna e Medicina Territoriale, Sapienza University of Rome. The patients with an abnormal Pap smear underwent colposcopy as a second level test and were subsequently divided, according to the colposcopy results, in two groups. The first (Group 1) was composed of 96 patients that had a positive colposcopy (39 ASC-US and 57 LSILs), and the second (Group 2) was composed of 95 patients with a negative satisfactory colposcopy (45 ASC-US and 50 LSILs). The cytological exams were reported according to the Bethesda System 2001 (7). Biopies were classified according to CIN classification.

The age of the women ranged from 18 to 54 years (average 38) in the ASC-US group, and from 18 to 50 years (average 34) in the LSIL group.

Sampling for p16INK4a was performed in a liquid base medium (Thin Prep, Hologic Italia, Rome, Italy) during colposcopy. When a lesion was evident, a biopsy was performed.

Ninety-five patients (45 ASC-US and 50 LSILs) without any colposcopic evident lesion, and therefore without any biopsy, were followed up for 1 year through cytology and colposcopy. The cytological results and the results of the biopsy performed when a lesion appeared were correlated with the p16INK4a status at enrollment.

For p16INK4a immunocytochemical analysis, a commercially available monoclonal antibody (clone E6H4, CINtec p16INK4a; MTM Laboratories, Heidelberg, Germany) was used. The procedure was performed according to the standard procedure. A positive p16INK4 result was defined by immunostaining of at least one morphologically identified abnormal (dysplastic or atypical) cell. Staining intensity and signal localization were not factors in determining a positive p16INK4a result. A negative p16INK4a result was defined as no cell staining or staining of morphologically normal metaplastic or endocervical cells, according to the manufacturer's instructions (19).

Informed consent of all of the patients was obtained. The Chi-square test was used for statistical analysis.
Table I. Correlation between cytology and p16INK4a.

\begin{tabular}{llrrrr}
\hline & \multicolumn{2}{c}{ ASC-US } & & \multicolumn{2}{c}{ LSILs } \\
\cline { 2 - 3 } \cline { 5 - 6 } & No. & $\%$ & & No. & $\%$ \\
\hline p16INK4a-negative & 51 & 60.7 & & 64 & 59.8 \\
p16INK4a-positive & 33 & 39.3 & & 43 & 40.2 \\
Total & 84 & 100.0 & & 107 & 100.0 \\
\hline
\end{tabular}

\section{Results}

One hundred and ninety-one patients were selected for this study and presented with 84 ASC-US and 107 LSILs as identified by cytology.

Thirty-three (39.3\%) (CI 95\% 28.9-49.7) of the ASC-US patients and $43(40.2 \%)$ (CI 95\% 30.9-49.5) of the LSIL patients were positive for p16INK4a (Table I). p16INK4a positivity was not statistically different between the ASC-US and LSIL patients.

The histological results of the Group 1 patients are reported in Tables II and III.

Fifty-nine (61\%) of the 96 patients that underwent biopsy were p16INK4a-negative (27 ASC-US and 32 LSILs). The histology of the 27 p16INK4a-negative ASC-US patients consistently showed a low-grade lesion (24 condilomas and 3 CIN1) (Table II). Of the 32 p16INK4a-negative LSIL patients, 30 presented with a lesion <CIN2 (6 metaplasia, 16 condilomas and 8 CIN1), while only 2 had CIN2 (Table III).

Thirty-seven of the $96(39 \%)$ Group 1 patients were positive for p16INK4a (12 ASC-US and 25 LSILs). The biopsy of 6 out of the 12 ASC-US patients with a positive p16INK4a revealed a CIN2 lesion (Table II). Biopsy of the 25 p16INK4a-positive LSIL patients revealed a condiloma in 8 patients, a CIN1 in 4 patients, a CIN2 in 10 patients and a CIN3 in 3 (Table III).

In the Group 1 cases, the p16INK4a test had $100 \%$ sensitivity for CIN2+ in the ASC-US patients and $87 \%$ sensitivity in the LSIL patients; the specificity of the test for CIN2+ was $82 \%$ in ASC-US and $71 \%$ in LSIL cases. The positive predictive value (PPV) was $50 \%$ in the ASC-US and $52 \%$ in the LSIL cases. The negative predictive value (NPV) was $100 \%$ for ASC-US and $94 \%$ for LSIL.

In Group 1 ASC-US cases, $82 \%$ of the CIN1/condilomas $(<\mathrm{CIN} 2)$ were p16INK4a-negative and all CIN2 were p16INK4a-positive ( $\mathrm{p}=0.00044)$. In Group $1 \mathrm{LSIL}$ cases, $71 \%$ of lesions <CIN2 were p16INK4a-negative and $87 \%$ of CIN2/3 were p16INK4a-positive ( $\mathrm{p}=0.00033)$.

Group 2 was composed of 95 (45 ASC-US and 50 LSILs) patients with no evident lesions at enrollment. Of these, 62 were p16INK4a-negative (30 ASC-US and 32 LSILs) and 33 were p16INK4a-positive (15 ASC-US and 18 LSILs). In 26 out of the 95 patients, a lesion became visible and, therefore, a biopsy was performed during the follow-up period.

All p16INK4a-negative ASC-US (30/30) and 25 out of 32 p16INK4a-negative LSIL patients had a negative Pap test and colposcopy within the 1-year follow-up (Tables IV and $\mathrm{V})$. The remaining 7 LSIL patients with negative p16INK4a at enrollment had a positive Pap test and colposcopic exam 
Table II. Correlation between histology and p16INK4a in patients with ASC-US (Group 1).

\begin{tabular}{|c|c|c|c|c|c|c|c|c|}
\hline & \multicolumn{4}{|c|}{$<\mathrm{CIN} 2$} & \multicolumn{2}{|c|}{ CIN2+ } & & \\
\hline & \multicolumn{2}{|c|}{ Condiloma } & \multicolumn{2}{|c|}{ CIN1 } & \multicolumn{2}{|c|}{ CIN2 } & \multicolumn{2}{|c|}{ Total } \\
\hline & No. & $\%$ & No. & $\%$ & No. & $\%$ & No. & $\%$ \\
\hline p16INK4a-negative & 24 & 88.9 & 3 & 11.1 & - & & 27 & 100 \\
\hline p16INK4a-positive & 3 & 25.0 & 3 & 25.0 & 6 & 50.0 & 12 & 100 \\
\hline Total & 27 & & 6 & & 6 & & 39 & \\
\hline
\end{tabular}

Statistical analysis for $<$ CIN2 vs. CIN2+ $(\mathrm{p}=0.00044)$.

Table III. Correlation between histology and p16INK4a in patients with LSIL (Group 1).

\begin{tabular}{|c|c|c|c|c|c|c|c|c|c|c|c|c|}
\hline & \multicolumn{6}{|c|}{$<\mathrm{CIN} 2$} & \multicolumn{4}{|c|}{ CIN2+ } & & \\
\hline & \multicolumn{2}{|c|}{$\begin{array}{c}\text { Negative } \\
\text { (metaplasia) }\end{array}$} & \multicolumn{2}{|c|}{ Condiloma } & \multicolumn{2}{|c|}{ CIN1 } & \multicolumn{2}{|c|}{ CIN2 } & \multicolumn{2}{|c|}{ CIN3 } & \multicolumn{2}{|c|}{ Total } \\
\hline & No. & $\%$ & No. & $\%$ & No. & $\%$ & No. & $\%$ & No. & $\%$ & No. & $\%$ \\
\hline p16INK4a-negative & 6 & 18.75 & 16 & 50.0 & 8 & 25.0 & 2 & 6.25 & - & & 32 & 100 \\
\hline p16INK4a-positive & - & & 8 & 32.0 & 4 & 16.0 & 10 & 40.0 & 3 & 12 & 25 & 100 \\
\hline Total & 6 & & 24 & & 12 & & 12 & & 3 & & 57 & \\
\hline
\end{tabular}

Statistical analysis for $<\mathrm{CIN} 2 \mathrm{vs}$. CIN2 $+(\mathrm{p}=0.00033)$.

Table IV. Cytological and histological follow-up (F-U) of ASC-US patients (Group 2).

\begin{tabular}{|c|c|c|c|c|c|c|}
\hline & \multicolumn{2}{|c|}{ Negative F-U } & \multicolumn{2}{|c|}{ Positive F-U } & \multicolumn{2}{|c|}{ Total } \\
\hline & No. & $\%$ & No. & $\%$ & No. & $\%$ \\
\hline p16INK4a-negative & 30 & 100 & - & & 30 & 100 \\
\hline p16INK4a-positive & 9 & 60 & $6^{\mathrm{a}}$ & 40 & 15 & 100 \\
\hline Total & 39 & & 6 & & 45 & \\
\hline
\end{tabular}

${ }^{a}$ All the six positive cases were LSIL and CIN1 lesions. Statistical analysis for positive vs. negative F-U (p=0.00113).

during follow-up; the biopsy revealed 4 condilomas, 1 CIN1, 2 CIN2 and no CIN3 (Table V).

Six out of the 15 p16INK4a-positive ASC-US patients had a positive Pap test (LSIL) within the 1-year follow-up; all lesions were histologically confirmed as low grade (Table IV).

Thirteen out of the 18 LSIL patients with positive p16INK4a at enrollment had a positive Pap test and colposcopic exam during follow-up; 7 of them (54\%) revealed a high-grade histologic lesion (5 CIN2 and 2 CIN3) (Table V).

In the Group 2 subjects, the p16INK4a test had $100 \%$ sensitivity for CIN2+ in the ASC-US patients and 65\% sensitivity in the LSIL patients. The specificity of the test for CIN2+ was $77 \%$ in ASC-US and $83 \%$ in LSIL cases. The PPV was $40 \%$ in the ASC-US and $72 \%$ in the LSIL cases, while the NPV was $100 \%$ for ASC-US and $78 \%$ for LSIL cases.

In Group 2 ASC-US cases, $77 \%$ of the patients with a negative follow-up were p16INK4a-negative at enrollment, while all patients with a positive follow-up were p16INK4a-positive $(\mathrm{p}=0.00113)$.

In Group 2 LSIL cases, during the same follow-up period, $83 \%$ of the patients with a negative follow-up were p16INK4anegative at enrollment, while $65 \%$ of the patients with a positive follow-up were p16INK4a-positive ( $\mathrm{p}=0.0014)$. In fact, $39 \%$ of the p16INK4a-positive LSIL patients had high-grade histological lesions (CIN2-CIN3) vs. only $6 \%$ (CIN2) of the p16INK4a-negative LSIL patients of the same group. These statistically significant results indicated a strong association between the p16INK4a status and the follow-up outcome.

\section{Discussion}

A meta-analysis by Tsoumpou et al regarding the results of the p16INKa immunocytochemical test showed an average positivity of $45 \%$ both in ASC-US and in LSIL cases (20). Our 
Table V. Cytological and histological follow-up (F-U) of LSIL patients (Group 2).

\begin{tabular}{|c|c|c|c|c|c|c|c|}
\hline & \multicolumn{2}{|c|}{ Negative F-U } & \multicolumn{2}{|c|}{ Positive F-U } & \multirow[t]{2}{*}{ Histological results } & \multicolumn{2}{|c|}{ Total } \\
\hline & No. & $\%$ & No. & $\%$ & & No. & $\%$ \\
\hline \multirow[t]{2}{*}{ p16INK4a-negative } & 25 & 78.1 & 6 LSIL & 18.8 & $\begin{array}{l}4 \text { Condilomas } \\
1 \text { CIN1 } \\
1 \text { CIN2 }\end{array}$ & 32 & 100 \\
\hline & & & $1 \mathrm{HSIL}$ & 3.1 & $1 \mathrm{CIN} 2$ & & \\
\hline \multirow[t]{2}{*}{ p16INK4a-positive } & 5 & 27.8 & 8 LSIL & 44.4 & $\begin{array}{l}2 \text { Condilomas } \\
4 \text { CIN1 } \\
2 \text { CIN2 }\end{array}$ & 18 & 100 \\
\hline & & & 5 HSIL & 27.8 & $\begin{array}{l}3 \text { CIN2 } \\
2 \text { CIN3 }\end{array}$ & & \\
\hline
\end{tabular}

Total

30

20

50

Statistical analysis for positive vs. negative F-U ( $\mathrm{p}=0.0014)$.

present results were similar to the literature data $(39.3 \%$ of ASC-US and $40.2 \%$ of LSIL cases).

Our data indicated that all of the p16INK4a-negative ASC-US cases and $94 \%$ of the p16INK4a-negative LSIL patients who underwent biopsy corresponded histologically to metaplasia or low-grade lesions (NPV for CIN2+ of $100 \%$ in ASC-US and 94\% in LSIL cytology) with no detected CIN3 lesions. These results confirm the high-negative predictive value of p16INK4a, as previously pointed out in studies by Hariri and Oster (21) and Rocha et al (22). In addition, we substantiated that such a negative predictive value can also be extended to the 1-year follow-up for patients who have positive cytology and negative colposcopy at enrollment (NPV for CIN2+ of $100 \%$ for ASC-US patients and NPV for CIN2+ of $78 \%$ for LSIL patients after a 1-year period). Thus, p16INK4a may play an important role in the diagnostic-prognostic management of p16INK4a-negative ASC-US patients; in fact, these patients not only did not have high-grade intraepithelial lesions at the initial evaluation, but also did not develop them during the 1-year follow-up.

The PPV was not as high as the NPV. However, $50 \%$ of the positive ASC-US and 52\% of the p16INK4a-positive LSILs (with a positive colposcopy) were correlated with histologically confirmed high-grade intraepithelial lesions. Only $40 \%$ of the ASC-US, but $72 \%$ of the p16INK4a-positive LSIL patients (without colposcopic evident lesions at enrollment) had a positive Pap test within 1 year, and 39\% of the latter had histologically confirmed high-grade lesions. Thus, the cytomorphological aspect of a low-grade squamous intraepithelial lesion, in association with positive p16INK4a, indicates the need for strict follow-up of these patients $(21,23)$. Negri et al identified p16INK4a as a marker for the risk of progression, by determining that p16INK4a was positive in $74.2 \%$ of CIN1 that had progressed to CIN3 (23).

Our study confirmed the premise that p16INK4a positivity is a marker of high-grade histological intraepithelial disease of the uterine cervix. The identification of the overexpression of p16INK4a, a regulatory protein of the cellular cycle, in fact, is able to identify those women who have an underlying high-grade cervical intraepithelial neoplasia in the subgroup of patients with HR HPV ASC-US or LSIL cytology, allowing the stratification of such patients in different risk groups. This can be explained by the fact that p16INK4a expression offers insight into the biology of the lesion, whereas cytology is limited to an evaluation of the surface of the epithelium at a particular moment, and may not show the higher grade lesions that may be present in the underlying epithelial layers $(24,25)$. In fact, p16INK4a has been used in the triage to identify high-grade cervical intraepithelial neoplasia in women with ASC-US and LSIL cytology (26).

On the other hand, the two p16INK4a-negative CIN2 at enrollment (Table III) and the two p16INK4a-negative CIN2 at follow-up (Table V) may be interpreted as intraepithelial cervical lesions diagnosed at a particular moment of their natural history. These lesions may represent a biotype more similar to low-grade lesions rather than lesions that progress to CIN3.

There is increasing awareness that not all CIN2 lesions are 'pre-cancer'. A significant portion of CIN2 lesions represents regressive lesions. It is estimated that approximately $40 \%$ of these lesions regress over a 2 -year period $(27,28)$. The regression is more likely in young women under 30 years of age, whose lesions involve only one cervical quadrant with type one transformation zone, without any colposcopic sign of severity, and whose cervical smear and biopsy results are concordant (28). It is also worth mentioning that CIN2 is the least reproducible of all cervical diagnoses that have a high inter- and intra-observer variability (29). In fact, p16INK4a may be a useful marker for a more precise biological classification of these 'transient lesions' (CIN2). To substantiate this, Bergeron et al demonstrated that p16INK4a significantly increased the inter-observer concordance and the diagnostic accuracy of high-grade intraepithelial neoplasia (30). In addition, immunohistochemical staining for p16INK4a was found to be a useful and reliable diagnostic adjuvant in CIN2+ histology $(31,32)$ and in cases of a discrepancy between a positive Pap test and a negative biopsy (33). 
In conclusion, a negative result for p16INK4a is, consistently, a marker of the absence of a high-grade CIN. Because of this high-negative predictive value, demonstrated both in the literature (21,33-35) and by our data, particularly in ASC-US cytology, the p16INK4a test should be included in the clinical diagnostic flow chart in order to identify patients with low or no risk of progression.

The introduction of an additional new test for the management of an abnormal Pap test may increase costs, yet it may decrease costs by prolonging follow-up intervals, avoiding unnecessary exams and reducing possible over-treatment. Management of the p16INK4a test and its clinical use should not, however, be left to the choice of a 'general' clinician, as this could result in a multitude of unnecessary tests. Patients must be directed to second level centers for the correct management of the disease.

We must, however, point out that the colposcopic exam remains the milestone in the management of an abnormal Pap test and, independently from the results of the p16INK4a, it allows visualization of a small group of negative p16INK4a high-grade cervical lesions that could follow different cancerogenetic pathways (36).

Our data confirm that for the clinical management of preneoplastic pathology of the uterine cervix it is necessary to acquire not only phenotypical data (by cytology, colposcopy and histology), but also bio-molecular data (p16INK4a). This allows an image of a lesion with a higher level of accuracy at a specific moment of its natural history.

In conclusion, p16INK4a, especially its NPV, may be used as an integrated diagnostic tool with added prognostic value in the prediction of the clinical outcome of cervical preneoplastic lesions.

\section{Acknowledgements}

The authors acknowledge Ms. Maria Maddalena Barbieri for the statistical interpretation of the data.

\section{References}

1. Zur Hausen H: Papillomavirus infections: a major cause of human cancers. Biochim Biophys Acta 1288: F55-F78, 1996.

2. Mitchell MF, Tortolero-Luna G, Wright T, Sarkar A Richards-Kortum R, Hong WK and Schottenfeld D: Cervical human papillomavirus infection and intraepithelial neoplasia: a review. J Natl Cancer Inst Monogr 21: 17-25, 1996.

3. Ostor AG: Natural history of cervical intraepithelial neoplasia: a critical review. Int J Gynecol Pathol 12: 186-192, 1993.

4. Wright TC Jr, Massad LS, Dunton CJ, Spitzer M, Wilkinson EJ and Solomon D: 2006 American Society for Colposcopy and Cervical Pathology-sponsored Consensus Conference. 2006 consensus guidelines for the management of women with abnormal cervical cancer screening tests. Am J Obstet Gynecol 197: 346-355, 2007.

5. Gestione della paziente con Pap test anormale: Linee guida 2006 a cura della Società Italiana di Colposcopia e Patologia Cervico Vaginale (SICPCV). 1st edition. Articoli Editore. Modena 21: $2-26,2006$

6. Jordan J, Martin-Hirsch P, Arbyn M, Schenck U, Baldauf JJ, da Silva D, Anttila A, Nieminen P and Prendiville W: European guidelines for clinical management of abnormal cervical cytology, Part 2. Cytopathology 20: 5-16, 2009.

7. Solomon D, Davey D, Kurman R, Moriarty A, O'Connor D, Prey M, Raab S, Sherman M, Wilbur D, Wright T Jr and Young N; Forum Group Members; Bethesda 2001 Workshop: The 2001 Bethesda system: terminology for reporting results of cervical cytology. JAMA 287: 2114-2119, 2002.
8. Solomon D, Schiffman M, Tarone R; ALTS Group: Comparison of three management strategies for patients with atipical squamous cells of undetermined significance: baseline results from a randomized trial. J Natl Cancer Inst 93: 293-299, 2001.

9. ASCUS-LSIL Triage study Group: Results of a randomized trial on the management of cytology interpretations of atypical squamous cells of undetermined significance. Am J Obstet Gynecol 188: 1382-1392, 2003.

10. Ortega S, Malumbres M and Barbacid M: Cyclin D-dependent kinases, INK4 inhibitors and cancer. Biochim Biophys Acta 1602: 73-87, 2002.

11. Yugawa T and Kiyono T: Molecular mechanisms of cervical carcinogenesis by high-risk human papillomaviruses: novel functions of E6 and E7 oncoproteins. Rev Med Virol 19: 97-113, 2009.

12. Khleif SN, DeGregori J, Yee CL, Otterson GA, Kaye FJ, Nevins JR and Howley PM: Inhibition of cyclin D-CDK4/CDK6 activity is associated with an E2F-mediated induction of cyclin kinase inhibitor activity. Proc Natl Acad Sci USA 93: 4350-4354, 1996.

13. Klaes R, Friedrich T, Spitkovsky D, Ridder R, Rudy W, Petry U, Dallenbach-Hellweg G, Schmidt D and von Knebel Doeberitz M: Overexpression of p16(INK4A) as a specific marker for dysplastic and neoplastic epithelial cells of the cervix uteri. Int J Cancer 92: 276-284, 2001.

14. Von Knebel Doeberitz M: New markers for cervical dysplasia to visualise the genomic chaos created by aberrant oncogenic Papillomavirus infections. Eur J Cancer 38: 2229-2242, 2002.

15. Baak JP, Kruse AJ, Robboy SJ, Janssen EAM, van Diermen B and Skaland I: Dynamic behavioural interpretation of cervical intraepithelial neoplasia with molecular biomarkers. J Clin Pathol 59: 1017-1028, 2006.

16. Trunk MJ, Dallenbach-Hellweg G, Ridder R, Petry KU, Ikenberg $\mathrm{H}$, Schneider V and von Knebel Doeberitz M: Morphologic characteristics of p16INK4a-positive cells in cervical cytology samples. Acta Cytol 48: 771-782, 2004.

17. Dehn D, Torkko KC and Shroyer KR: Human papillomavirus testing and molecular markers of cervical dysplasia and carcinoma. Cancer Cytopathol 111: 1-14, 2007.

18. Schledermann D, Andersen BT, Bisgaard K, Dohse M, Ejersbo D, Hoelund B, Horal P, Lindh M and Ryd W: Are adjunctive markers useful in routine cervical cancer screening? Application of ThinPrep samples with histological follow-up. Diagn Cytopathol 36: 453-459, 2008.

19. Manual CINtec MTM: http://www.mtmlabs.com.

20. Tsoumpou I, Arbyn M, Kyrgiou M, Wentzensen N, Koliopoulos G, Martin-Hirsch P, Malamou-Mitsi V and Paraskevaidis E: p16(INK4a) immunostaining in cytological and histological specimens from the uterine cervix: a systematic review and meta-analysis. Cancer Treat Rev 35: 210-220, 2009.

21. Hariri J and Oster A: The negative predictive value of p16INK4a to assess the outcome of cervical intraepithelial neoplasia in the uterine cervix. Int J Gynecol Pathol 26: 223-228, 2007.

22. Rocha AS, Bozzetti MC, Kirschnick LS and Edelweiss M: Antibody anti-p16 (INK4a) in cervical cytology. Acta Cytol 53: 253-262, 2009.

23. Negri G, Vittadello F, Romano F, Kasal A, Rivasi F, Girlando S, Mian C and Egarter-Vigl E: P16INK4a expression and progression risk of low-grade intraepithelial neoplasia of the cervix uteri. Virchows Arch 445: 616-620, 2004.

24. Alsharif M, Kjeldahl K, Curran C, Miller S, Gulbahce HE and Pambuccian SE: Clinical significance of the diagnosis of low-grade squamous intraepithelial lesion, cannot exclude highgrade squamous intraepithelial lesion. Cancer Cytopathol 117: 92-100, 2009

25. Al-Nourhji O, Beckmann MJ, Markwell SJ and Massad LS: Pathology correlates of a Papanicolaou diagnosis of low-grade squamous intraepithelial lesion, cannot exclude high-grade squamous intraepithelial lesion. Cancer 114: 469-473, 2008.

26. Wentzensen N, Bergeron C, Cas F, Vinokurova S and von Knebel Doeberitz M: Triage of women with ASCUS and LSIL cytology: use of qualitative assessment of p16INK4apositive cells to identify patients with high-grade cervical intraepithelial neoplasia. Cancer 111: 58-66, 2007.

27. Castle PE, Schiffman M, Wheeler C and Solomon D: Evidence for frequent regression of cervical intraepithelial neoplasiagrade 2. Obstet Gynecol 113: 18-25, 2009.

28. Carcopino X, Muszynski C, Merfui J-L, Gondry J and Boubli L: Should CIN2 and 3 be treated the same way? Gynecol Obstet Fertil 39: 94-99, 2011. 
29. Stoller MH and Schiffman M: Interobserver reproducibility of cervical cytology and histologic interpretations: realistic estimates from the ASCUS-LSIL Triage Study. JAMA 285: 1500-1505, 2001.

30. Bergeron C, Ordi J, Schmidt D, Trunk MJ, Keller T and Ridder R: European CINtec Histology Study Group. Conjunctive p16INK4a testing significantly increases accuracy in diagnosing high-grade cervical intraepithelial neoplasia. Am J Clin Pathol 133: 395-406, 2010.

31. Kurshumliu F, Thorns C and Gashi-Luci L: p16INK4A in routine practice as a marker of cervical epithelial neoplasia. Gynecol Oncol 115: 127-131, 2009.

32. Galgano MT, Castle PE, Atkins KA, Brix WK, Nassau SR and Stoler MH: Using biomarkers as objective standards in the diagnosis of cervical biopsies. Am J Surg Pathol 34: 1077-1087, 2010.

33. Monsonego J, Pollini G, Evrard MJ, Sednaoui P, Monfort L, Quinzat D, Dachez R and Syrjänen K: P16INK4a immunocytochemistry in liquid-based cytology samples in equivocal Pap smears: added value in management of women with equivocal Pap smear. Acta Cytol 51: 755-766, 2007.
34. Nieh S, Chen SF, Chu TY, Lai HC, Lin YS, Fu E and Gau CH: Is p16 (INK4A) expression more useful than human papillomavirus test to determine the outcome of atypical squamous cells of undetermined significance-categorized Pap smear? A comparative analysis using abnormal cervical smears with follow-up biopsies. Gynecol Oncol 97: 35-40, 2005.

35. Eleutério J Jr, Giraldo PC, Gonçalves AK, Cavalcante DI, de Almeida Ferreira FV, Mesquita SM and Morais SS: Prognostic markers of high-grade squamous intraepithelial lesions: the role of p16INK4a and high-risk human papillomavirus. Acta Obstet Gynecol Scand 86: 94-98, 2007.

36. Furtado YL, Almeida G, Lattario F, Silva KS, Maldonado P, Silveira FA, do Val IC, Fonseca R and Carvalho Mda G: The presence of methylation of the p16INK4A gene and human papillomavirus in high-grade cervical squamous intraepithelial lesions. Diagn Mol Pathol 19: 15-19, 2010. 